\title{
Intermittency and Anisotropy in the Ionized Interstellar Medium
}

\author{
Barney Rickett \\ Dept. of Electrical \& Computer Engineering, \\ University of California San Diego, La Jolla, CA 92093, USA \\ email: bjrickett@ucsd.edu
}

\begin{abstract}
The discovery of pulsars was closely followed by the discovery of dispersion and scattering in the interstellar plasma (ionized interstellar medium - IISM). The rich phenomena of scattering and scintillation have since been successfully modelled as propagation through a statistically uniform plasma turbulence with an isotropic Kolmogorov spectrum of density. However, this enticingly simple model fails to explain the many recent observations, that show anisotropic scattering from highly localized regions of the IISM often referred to as phase screens. I summarize the recent evidence from pulsars and also from very compact AGN sources, which can exhibit rapid scintillation and occasionally ESEs. The unknown astrophysical origin of these phenomena includes thin current sheets, the diffuse remnants of old supernova shells, and plasma filaments surrounding ubiquitous molecular clumps near young hot stars.
\end{abstract}

Keywords. pulsars: general, ISM: structure, scattering

\section{Early History}

In early 1968 Andrew Lyne and I were assigned observing time on the $76 \mathrm{~m}$ telescope at Jodrell Bank for our $\mathrm{PhD}$ research, respectively on lunar occultations and interplanetary scintillation. However the night before the observations were to begin, our supervisor Graham Smith received a call from Tony Hewish in Cambridge alerting him to the publication of the discovery paper (Hewish, Bell et al. 1968) and the coordinates of "CP1919". Though I was initially reluctant to chase a "Pulsating Radio Source"(!), the observations were extraordinary and very exciting; we were lucky to be at the start of pulsar astronomy which set the course for our subsequent research careers.

We had equipped the telescope to receive simultaneously at both 150 and $408 \mathrm{MHz}$, and for the pulsar "CP0328" we noticed that the pulse-to-pulse amplitude variations arrived later at $150 \mathrm{MHz}$ by about $5 \mathrm{sec}$. This dispersive delay proved that pulse-to-pulse variations are imposed at the source - i.e. intrinsic. In striving for better time resolution, we resoldered ever-smaller capacitors into the time constant circuits and decreased the IF bandwidths to minimise dispersion broadening. A side effect we noticed was a slow fading in pulse amplitude that became deeper in narrower bandwidths. This became my obsession, as we investigated the next 10 pulsars that were discovered. The fading was amazingly narrow in bandwidth, becoming narrower still for higher DM pulsars, giving the first observational evidence for interstellar scintillation(ISS) (Rickett 1969). In that paper I published the first dynamic spectrum of ISS, though it was recorded by Andrew, who gated the auto-correlation spectrometer in synchronism with the pulses.

Though I had been reading up on interplanetary scintillation, it was Scheuer's (1968) paper on interstellar scintillation that laid out the theory and its relation to observations. I applied his Gaussian model for the spectrum of interstellar electron density $\left(n_{e}\right)$ to our Jodrell observations and estimated $r_{o} \sim 10^{9} \mathrm{~m}$ for the characteristic scale of the 
Table 1. Evidence for Intermittency and Anisotropy in the Ionised Interstellar Medium

\begin{tabular}{|c|c|c|c|}
\hline Intermittency & Reference & Anisotropy & Reference \\
\hline Patchy $\mathrm{H}-\alpha$ maps & Finkbeiner (2003) & | "Streaks" in $\mathrm{H}-\alpha$ maps & Finkbeiner(2003) \\
\hline $\begin{array}{c}\text { Discrete DM events } \\
- \\
-\end{array}$ & $\begin{array}{l}\text { Coles et al. }(2015) \\
\text { Lam et al. }(2016) \\
\text { Jones et al. }(2017)\end{array}$ & & \\
\hline Variable ISS parameters & $\begin{array}{c}\text { Bhat et al. }(1999) \\
\text { Ramachandran et al. }(2006)\end{array}$ & $\begin{array}{l}- \\
-\end{array}$ & \\
\hline $\begin{array}{l}\text { Reverse scintillation Arcs } \\
\text { Multiple Forward Arcs } \\
\text { Time-variable reverse } \\
\text { arcs in B } 1737+13\end{array}$ & $\begin{array}{c}\text { Hill et al. }(2006) \\
\text { Putney \& Stinebring (2005) } \\
\text { Hemberger \& Stinebring }(2008)\end{array}$ & $\begin{array}{l}\text { Scintillation Arcs } \\
\text { VLBI of Arcs } \\
\text { Arcs in B } 1737+13\end{array}$ & $\begin{array}{c}\text { Stinebring et al. }(2001) \\
\text { Brisken et al. }(2010) \\
\text { ibid. }\end{array}$ \\
\hline $\begin{array}{l}\text { Scattered pulses with fast } \\
\text { rise and slow decay }\end{array}$ & $\begin{array}{l}\text { Bhat et al. (2004) } \\
\text { eg J1853+0545 }\end{array}$ & $\begin{array}{l}- \\
-\end{array}$ & \\
\hline $\begin{array}{c}\text { Faster increase in } \tau_{\text {scatt }} \\
\text { than } D M^{2.2}\end{array}$ & $\begin{array}{c}\text { Sutton (1971) } \\
\text { Bhat, Cordes, et al. (2004) }\end{array}$ & - & \\
\hline $\begin{array}{c}\text { Slower increase in } \tau_{\text {scatt }} \\
\operatorname{than} \lambda^{4}\end{array}$ & $\begin{array}{c}\text { Loehmer (2004) } \\
\text { Bhat, Cordes, et al. }(2004)\end{array}$ & - & \\
\hline $\begin{array}{l}\text { AGN flux varying on } \\
\text { times }<1 \text { d (IDV) }\end{array}$ & $\begin{array}{l}\text { Kedziora-Chudczer (1997) } \\
\text { e.g. PKS } 0405-385\end{array}$ & $\begin{array}{l}\text { Annual variation } \\
\text { in IDV timescale }\end{array}$ & $\begin{array}{l}\text { Dennett-Thorpe } \\
\& \text { de Bruyn, } 2003\end{array}$ \\
\hline Extreme Scattering Events & Fiedler et al. (1987) & - & \\
\hline
\end{tabular}

nearby IISM (Rickett 1970). However, Salpeter (1969) pointed out that the Fresnel scale $\sqrt{Z \lambda / 2 \pi} \approx 2 \times 10^{9} \mathrm{~m}$ for scattering at a distance $Z \sim 300 \mathrm{pc}$, so if $n_{e}$ in the IISM had a power law spectrum the estimated $r_{o}$ was probably imposed by Fresnel diffraction.

\section{Intermittency and Anisotropy in Interstellar Turbulence}

Then Lee \& Jokipii (1976), taking ideas from turbulence in the solar wind plasma, proposed a uniform isotropic Kolmogorov spectrum for $n_{e}$ in the IISM. This idea survived (flourished) over the following decades, providing a framework for interpreting most ISS observations, see Rickett (1990) and Narayan (1992). In its simplest form the model assumes that the $3 \mathrm{D}$ spectrum of $n_{e}$ is a constant times wavenumber ${ }^{-11 / 3}$, with the constant being invariant throughout the Galactic disc. Armstrong et al. (1995) presented evidence in support of this very simplistic idea for the local region, out to about $1 \mathrm{kpc}$.

However, there are now many lines of evidence that the IISM is far from uniform (its turbulence is intermittent like "clear air turbulence" in the atmosphere) and that its small scale structure can be highly anisotropic. My goal here is to list the lines of evidence (Table 1) and also to list the variety of models (Table 2) for the, as yet unknown, underlying astrophysical cause of these plasma concentrations (screens).

Sutton (1971) had already noted evidence for non-uniformity in how the interstellar broadening time $\tau_{\text {scatt }}$ increases faster than $D M^{2}$, which is expected for statistically uniform scattering. This discrepancy is now very well documented for a large population of pulsars by Bhat et al. (2004). The underlying cause has to be increased patchiness (i.e. variance in $n_{e}$ ) for high $D M$ pulsars at low Galactic latitudes and toward the inner Galaxy. Patches of enhanced Kolmogorov turbulence have since been incorporated into most models of the Galactic distribution of electrons (Taylor \& Cordes 1993; Cordes \& Lazio 2001; Yao et al. 2017). However, there is little direct evidence that they are driven by turbulent motions. Indeed the enhanced scattering could due to plasma shocks.

The first entry in Table 1 is evidence from the all-sky maps of $\mathrm{H}-\alpha$ emission intensity $I_{\alpha}$, assembled by Finkbeiner (2003), which show high $I_{\alpha}(\propto E M)$ from low Galactic latitudes 
Table 2. Proposed Origins of Localized Anisotropic Scattering

\begin{tabular}{|c|c|c|}
\hline Model & Reference & Localized (L) or Anisotropy (A) \\
\hline Refraction from a Plasma Lens & Romani et al. (1988) & $\mathrm{L}(\mathrm{ESE})$ \\
\hline Scattering from localized Plasma Turbulence & Fiedler et al. (1987) & $\mathrm{L}(\mathrm{ESE})$ \\
\hline Refraction by ripples on a sheet of plasma & $\begin{array}{l}\text { Pen \& Levin }(2014) \\
\text { Simard \& Pen (2017) }\end{array}$ & $\begin{array}{l}\mathrm{A}, \mathrm{L}(\mathrm{Arcs}) \\
\mathrm{A}, \mathrm{L}(\operatorname{Arcs})\end{array}$ \\
\hline $\begin{array}{l}\text { Grazing transmission through shells } \\
\text { \& ionization fronts of molecular clouds }\end{array}$ & $\begin{array}{l}\text { Boldyrev \& Gwinn (2003) } \\
\text { Boldyrev \& Königl (2006) }\end{array}$ & $\begin{array}{l}\mathrm{A}, \mathrm{L}(\mathrm{Arcs}) \\
\mathrm{A}, \mathrm{L}(\operatorname{Arcs})\end{array}$ \\
\hline $\begin{array}{c}\text { Ionized shells of old supernova remnants } \\
-\end{array}$ & $\begin{array}{l}\text { Spitler \& Spangler (2005) } \\
\text { Clegg, Fey et al. }(1998)\end{array}$ & $\begin{array}{l}\text { A,L (limits) } \\
\text { A,L (limits) }\end{array}$ \\
\hline $\begin{array}{l}\text { Filaments ionized by Hot stars, from } \\
\text { molecular clumps similar to "Cometary Knots" }\end{array}$ & Walker et al. (2017) & $\begin{array}{c}\mathrm{A}, \mathrm{L} \\
(\mathrm{IDV}, \mathrm{ESE})\end{array}$ \\
\hline
\end{tabular}

within $\pm 20 \mathrm{deg}$, due to both star-forming HII regions and the debris from supernovae. Both are candidates for enhancced interstellar scattering. Recent years have seen superb pulse arrival time obsevations motivated by the search for gravitational waves. To correct for variations in the dispersive delay or scattering in the IISM, observers have monitored $D M$ from millisecond pulsars with great precision, resulting in excellent long data sets of the $D M$ that provide new insight into the IISM. The second entry in Table 1 lists several of these, which have shown both stochastic and systematic variations in $D M$. The systematic variations include gradients and occasional discrete $D M$ events, caused as the pulsar line of sight passes over a localized region of increased (or diminished) electron density. The variability in the scattering parameters, which too must be caused by discrete stucture scanned by the line of sight, is further evidence for patchiness.

Even though the uniform isotropic Komogorov model is often cited, many specific ISS measurements are modelled by scattering at a plasma phase screen, located somewhere between the observer and the source. For example, in the scintillation arc phenomenon discovered by Stinebring et al. (2001) there are often narrow parabolic traces of power in the secondary spectrum, which is the 2-D power spectrum of the ISS dynamic spectrum from a pulsar. The narrow (forward) parabolic form with an apex at the origin can only be explained by scattering at a localized screen (Cordes et al. 2006), in which an unscattered wave interferes with a distribution of waves that are scattered anisotropically along a particular angle on the sky. In contrast a broader parabolic form would be expected for scattering distributed all along the path from the pulsar. Thus arc observations provide evidence for turbulence that is both clumpy and anisotropic; see also more direct evidence by Brisken et al. (2010). Some nearby pulsars (B0834+06, B1737+13) also show reversed parabolic arclets with apexes along a forward arc. These are explained by centers of scattering (or refraction) that lie along a line on the sky and interfere with a broadened distribution extending anisotropically along the same line. The wavelength independence of the arclets requires the centers to be at fixed locations transverse to the line of sight offset by 1-10 AU and give further evidence for localized scattering.

Anisotropy is also needed to explain the annual modulation reported in the scintillation timescale of very compact AGN cores, which vary faster than a day (IDV) (Rickett et al. 2001) ; Dennett-Thorpe \& de Bruyn (2003) showed that the short timescales in $\mathrm{J} 1819+384$ also require very nearby scattering in the Local Interstellar Cloud.

There is still no consensus on the astrophysical origin of the localized anisotropic plasma structures, as evident from the range of phenomena listed in Table 2. Scales are on the order of an AU rather than a parsec. Note the distinction between deflections due 
to scattering and refraction (lensing). While scattering implies turbulence internal to the localized plasma structures, refraction does not. In both cases the mean $n_{e}$ implied from the observed angles of deflection are uncomfortably high, exceeding expectations from pressure balance. In both cases $n_{e}$ is reduced for near grazing incidence on sheet-like structures, which can be characterized by a width to depth ratio $A$. For example, Rickett (2007) estimated $n_{e}>100 / A \mathrm{~cm}^{-3}$ (lensing); $n_{e}>18 A^{-.5} \mathrm{~cm}^{-3}$ (scattering). The last entry in Table 2 is a striking new idea, that relates the screens to a hot star near enough to the line of sight to ionize filaments oriented radially outward from the star.

\section{References}

Armstrong, J. W., Rickett, B. J., \& Spangler, S. R. 1995, ApJ, 443, 209

Bhat, N. D. R., Gupta, Y., \& Rao, A. P. 1999, ApJ, 514, 259

Boldyrev, S. \& Gwinn, C. R. 2003, ApJ, 584, 791

Boldyrev, S. \& Königl, S. 2006, ApJ, 640, 344

Bhat, N. D. R. \& Cordes, J. M. et al. 2004, ApJ, 605, 759

Brisken, W. F., Macquart, J.-P. et al. 2010, ApJ, 708, 232

Clegg, A. W., Fey, A. L., \& Lazio, T. J. W. 1998, ApJ, 496, 253

Cordes, J. M. \& Lazio, T. J. W. 2001, ApJ, 549, 997

Cordes, J.M., Rickett, B.J., Stinebring, D.R. \& Coles, W.A. 2006 ApJ, 637, 346

Dennett-Thorpe, J. \& de Bruyn, A. G. 2003, A\& A, 404, 113

Fiedler, R. L., Dennison, B., Johnston, K. J., \& Hewish, A. 1987, Nature, 326, 675

Finkbeiner, D. P. 2003, ApJS, 146, 407

Gupta, Y. \& Rickett, B. J., Lyne 1994, MNRAS, 269, 1035

Hemberger, D. L. \& Stinebring, D. S. 2008, ApJ, 674, L37

Hewish, A., Bell, S.J., Pilkington, J.D.H., Scott, P.F. \& Collins, R.A. 1968 Nature, 217, 709

Hill, A. S. \& Stinebring, D. R. et al. 2005, ApJ, 619, L171

Jones, M. L. et al. 2017, ApJ, 841, 125

Kedziora-Chudczer, L. 2006, MNRAS, 369, 449

Lam, M. T. \& Cordes, J. M. et al. 2016, ApJ, 821, 66

Lee, L. C. \& Jokipii, J. R. 1976, ApJ, 206, 735

Narayan, R. 1992, Phil. Trans. Roy. Soc. A, 341, 151

Pen, U.-L. \& Levin, Y. 2014, MNRAS, 442, 3338

Ramachandran, R., Demorest, P., D. C. Backer, Cognard, I., \& Lommen, A. 2006, ApJ, 645, 303

Rickett, B. J. 1969, Nature, 221, 158

Rickett, B. J. 1970, MNRAS, 150, 67

Rickett, B. J. 1990, ARAA, 28, 561

Rickett, B. J., Witzel,A., et al. 2001, ApJ, 550, 11

Rickett, B. J. 2007, ASP-CS, 365, 207

Romani, R. W., Blandford, R. D., \& Cordes, J. M. 1988, Nature, 328, 324

Salpeter, E. E. 1969, Nature, 221, 31

Scheuer, P. A. G. 1968, Nature, 218, 920

Simard, D. \& Pen, Ue-Li 2017, ADS arXiv:1703.06855

Spitler, L.G. \& Spangler, S.R.2005, ApJ, 632, 932

Stinebring, D. R. \& McLaughlin, M. A., et al. 2001, ApJ, 549, L97

Sutton, J. M. 1971, MNRAS, 155, 51

Taylor, J. H. \& Cordes, J. M. 1993, ApJ, 411, 674

Walker, M. A. \& Tuntsov, A. V., et al. 2017, ApJ, 843, 15

Yao, J. M., Manchester, R. N., \& Wang, N. 2017, ApJ, 835, 29 